\title{
Phase Velocities and Attenuation Distances in the Ionosphere
}

\author{
D. R. Croley, Jr. \\ McDonnell Aircraft Corporation, St. Louis, Mo. \\ and \\ B. S. Tanenbaum \\ Case Institute of Technology, Cleveland, Ohio
}

(Received January 8, 1965)

\begin{abstract}
Plane wave phase velocities and attenuation distances in an infinite, homogeneous, partly ionized gas composed of an interacting mixture of neutral molecules, ions, and electrons in the presence of a uniform magnetic field are determined for three sets of plasma conditions corresponding to the 80,100 , and $300 \mathrm{~km}$ levels of the ionosphere. Propagation in the parallel direction and the perpendicular direction relative to the magnetic field in the frequency range from approximately $10^{-5} \mathrm{~Hz}$ to $10^{9} \mathrm{~Hz}$ is considered.
\end{abstract}

\section{Introduction}

Tanenbaum and Mintzer [1962, 1963] have studied the propagation of plane waves in an infinite, homogeneous, partly ionized gas composed of an interacting mixture of neutral molecules, ions, and electrons in the presence of a uniform magnetic field. This letter reports the application of the three fluid theory to gas conditions at the 80,100 , and $300 \mathrm{~km}$ levels of the ionosphere. Phase velocity and attenuation distances have been calculated on an EDP computer using the dispersion relations obtained by Tanenbaum and Mintzer. Calculations are made for two distinct cases: (1) transverse wave propagation parallel to the uniform magnetic field (figs. 1 and 2); and (2) coupled longitudinal and transverse wave propagation perpendicular to the uniform magnetic field (figs. 3 through 8). The characteristics of the curves differ significantly at each altitude in both cases. The phase velocity curves obtained using the $300 \mathrm{~km}$ data resemble figures 2 and 4 of Tanenbaum and Mintzer [1962], where collisional damping effects are ignored, while the curves using the 80 and $100 \mathrm{~km}$ data are decidedly different (due to the increasing importance of the collision terms in the dispersion relations as the altitude decreases from 300 to $80 \mathrm{~km}$ ).
Considering first transverse waves propagating parallel to the magnetic field, we show in figures 1 and 2 the phase velocity, $\omega / \operatorname{Re} k$, and attenuation distance, $(\operatorname{Im} k)^{-1}$, as calculated directly from the dispersion relation [Tanenbaum and Mintzer, 1962],

$$
k^{2} c^{2}=\omega^{2}-\omega_{e}^{2}\left[1-\frac{\omega_{c}^{e} \omega_{c}^{i}}{\omega^{2} f^{2}} \pm \frac{\omega_{c}^{e}}{\omega}+\frac{i \nu_{e}}{\omega}\right]^{-1},
$$

where

$$
f^{2}=\left(\nu_{i n}+\nu_{n i}-i \omega\right) /\left(\nu_{n i}-i \omega\right) .
$$

The $(+)$ refers to left circular polarization (LCP), and the (-) refers to right circular polarization (RCP). Values of temperature, ion and electron density, neutral density, and ion mass, as well as the important frequencies and velocities for the 80,100 , and $300 \mathrm{~km}$ levels are summarized in table 1 [Chapman, 1956; Cowling, 1945; Minzner, Champion, and Pond, 1959]. The range of frequencies shown may be academic, but serves to display the important features contained in the dispersion relations. 


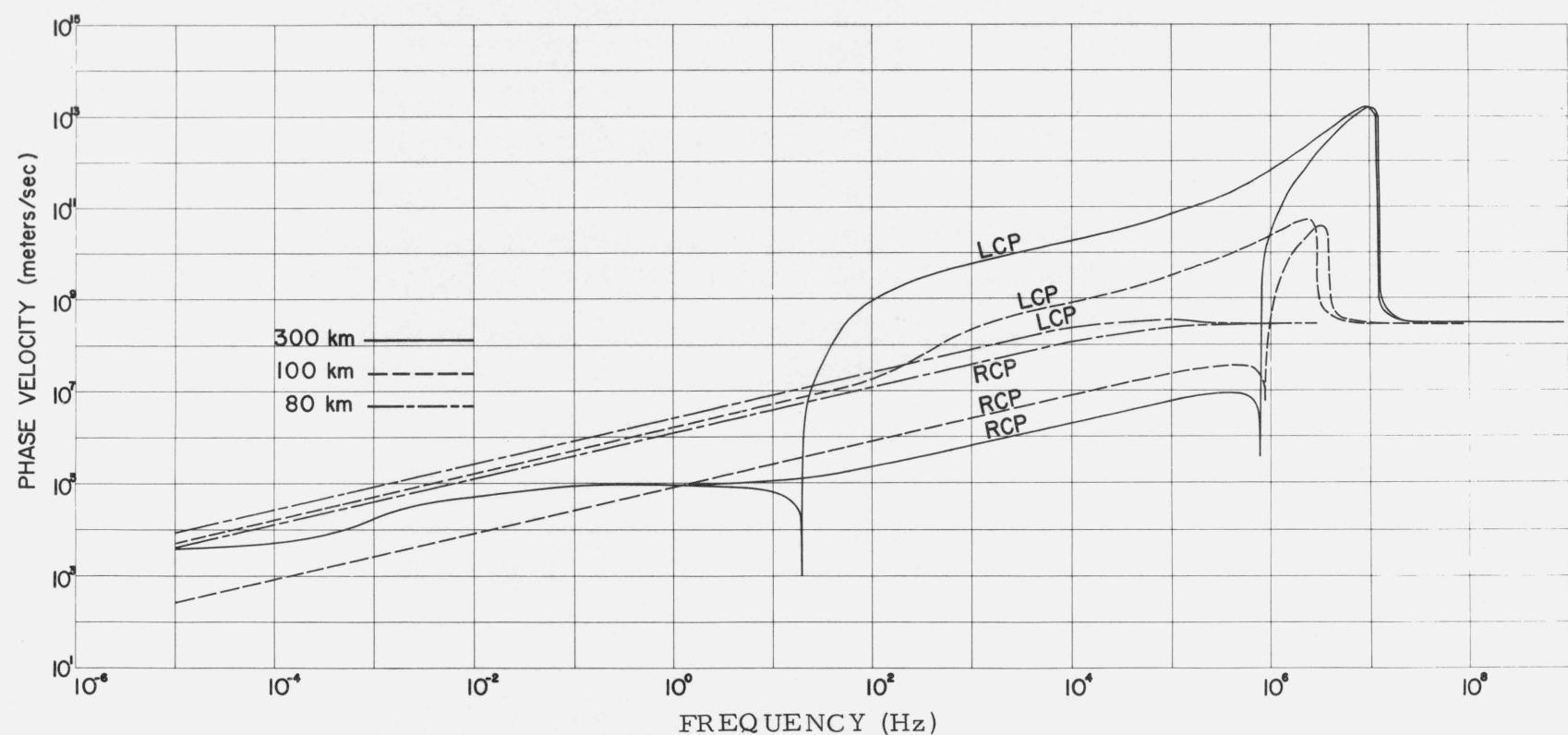

FiguRE 1. Phase velocities as a function of frequency for transverse waves traveling parallel to a uniform magnetic field at 80 , 100, and $300 \mathrm{~km}$.

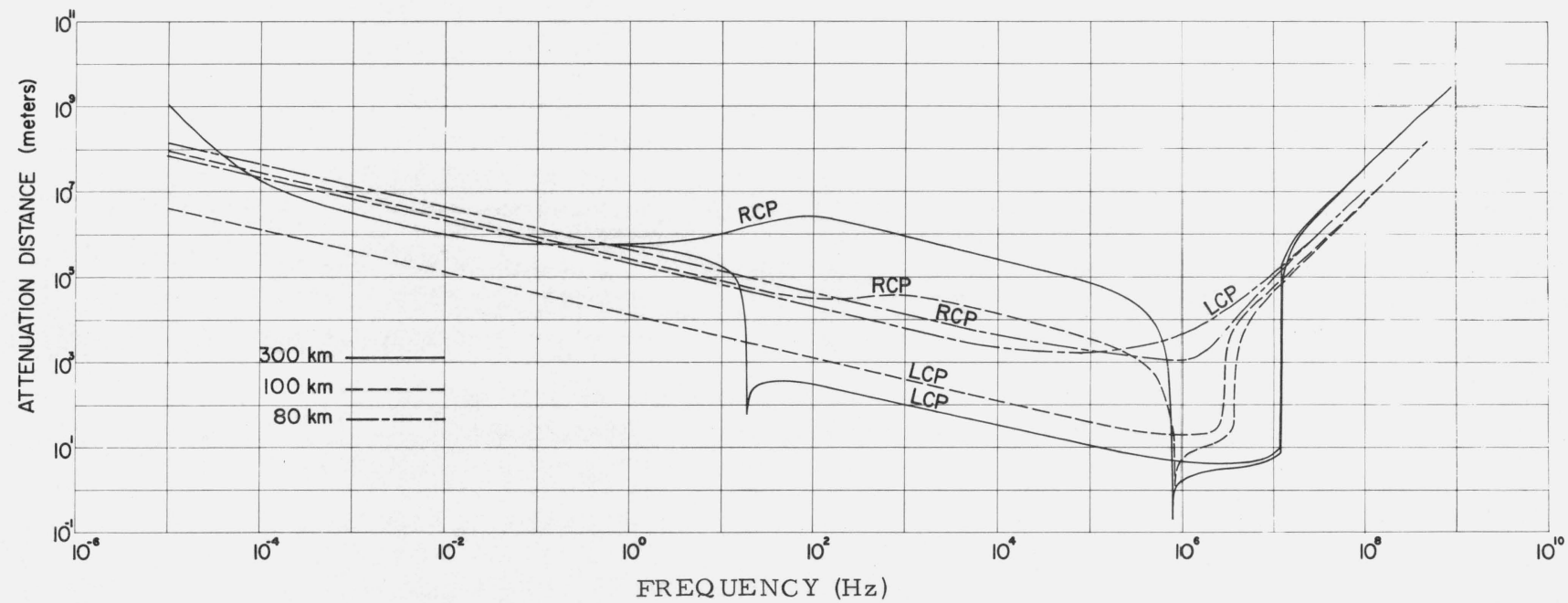

FIGURE 2. Attenuation distances as a function of frequency for waves traveling parallel to a uniform magnetic field at 80,100 , and $300 \mathrm{~km}$. 


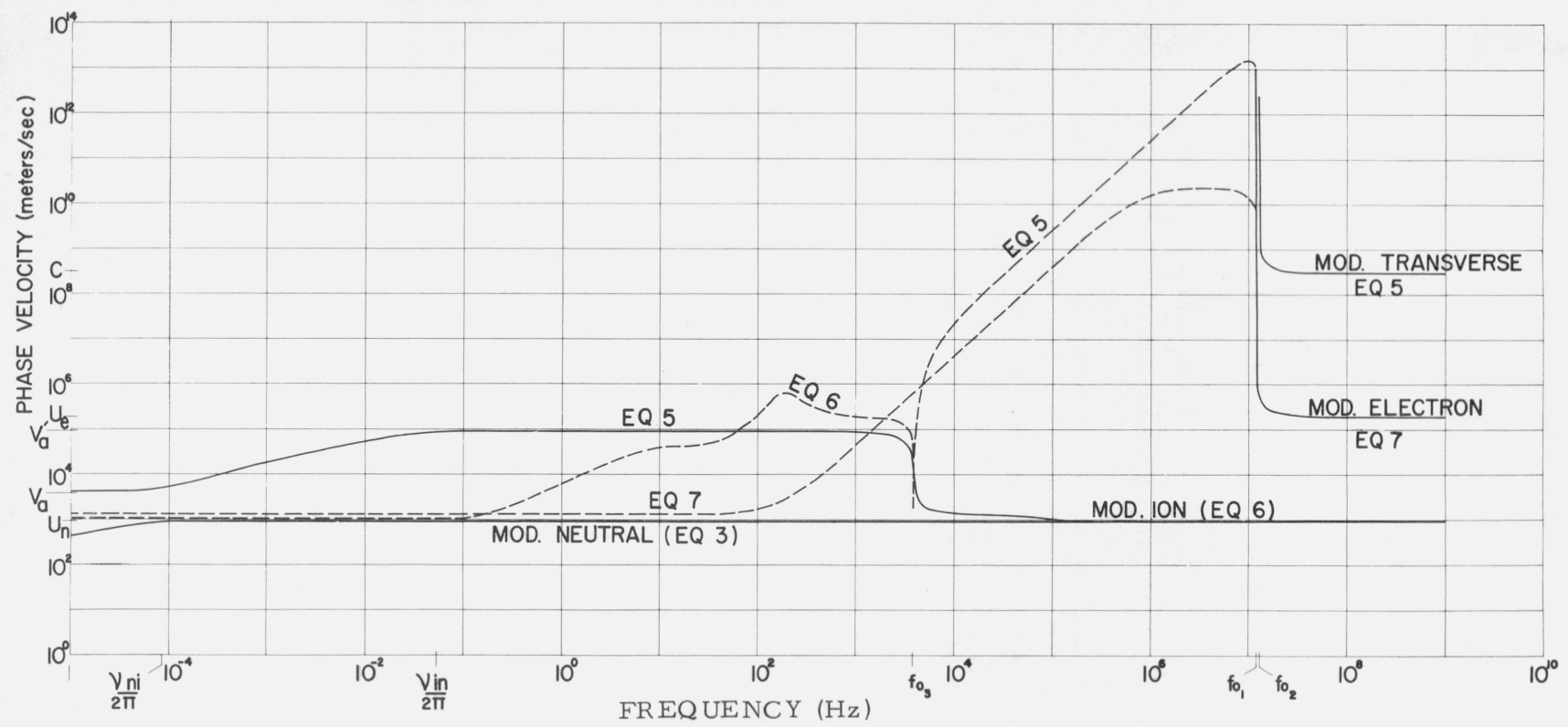

Figure 3. Phase velocities as a function of frequency for waves traveling perpendicular to a uniform magnetic field at $300 \mathrm{~km}$. The solid lines identify the wave modes described by Tanenbaum and Mintzer [1962]. The dotted lines are the strongly attenuated approximations.

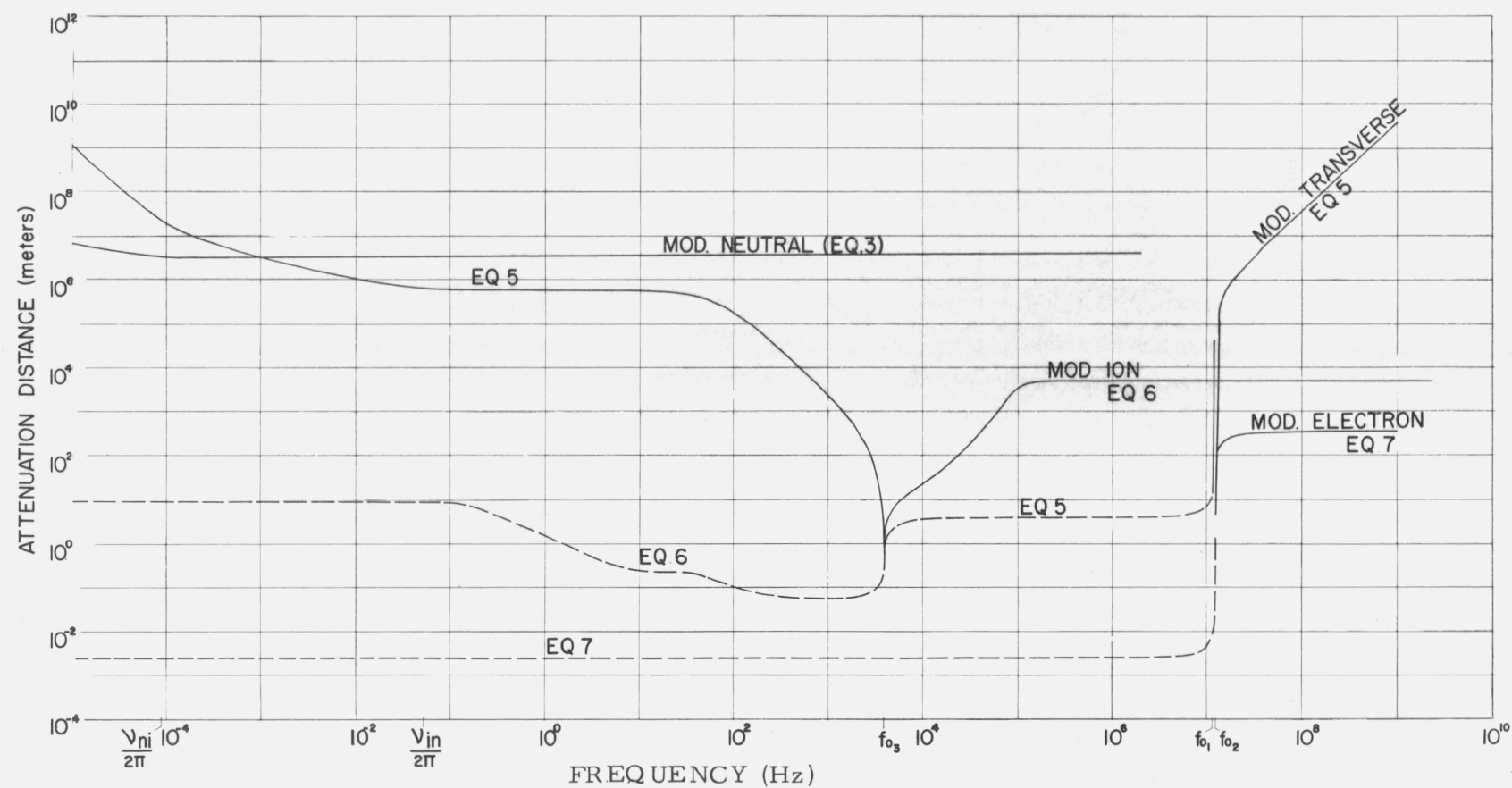

FigURE 4. Attenuation distances as a function of frequency for waves traveling perpendicular to a uniform magnetic field at $300 \mathrm{~km}$. 


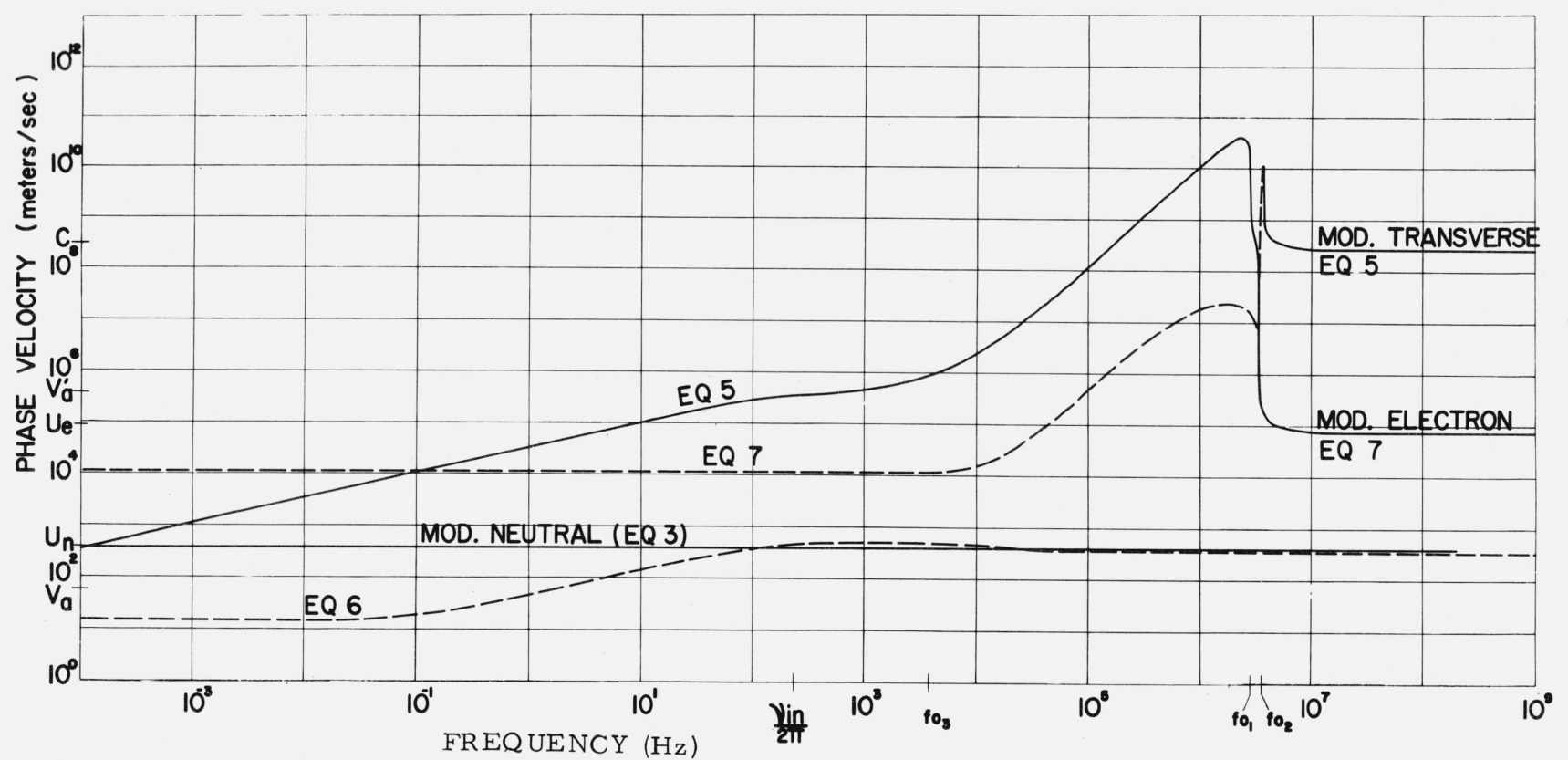

FigURE 5. Phase velocities as a function of frequency for waves traveling perpendicular to a uniform magnetic field at $100 \mathrm{~km}$.

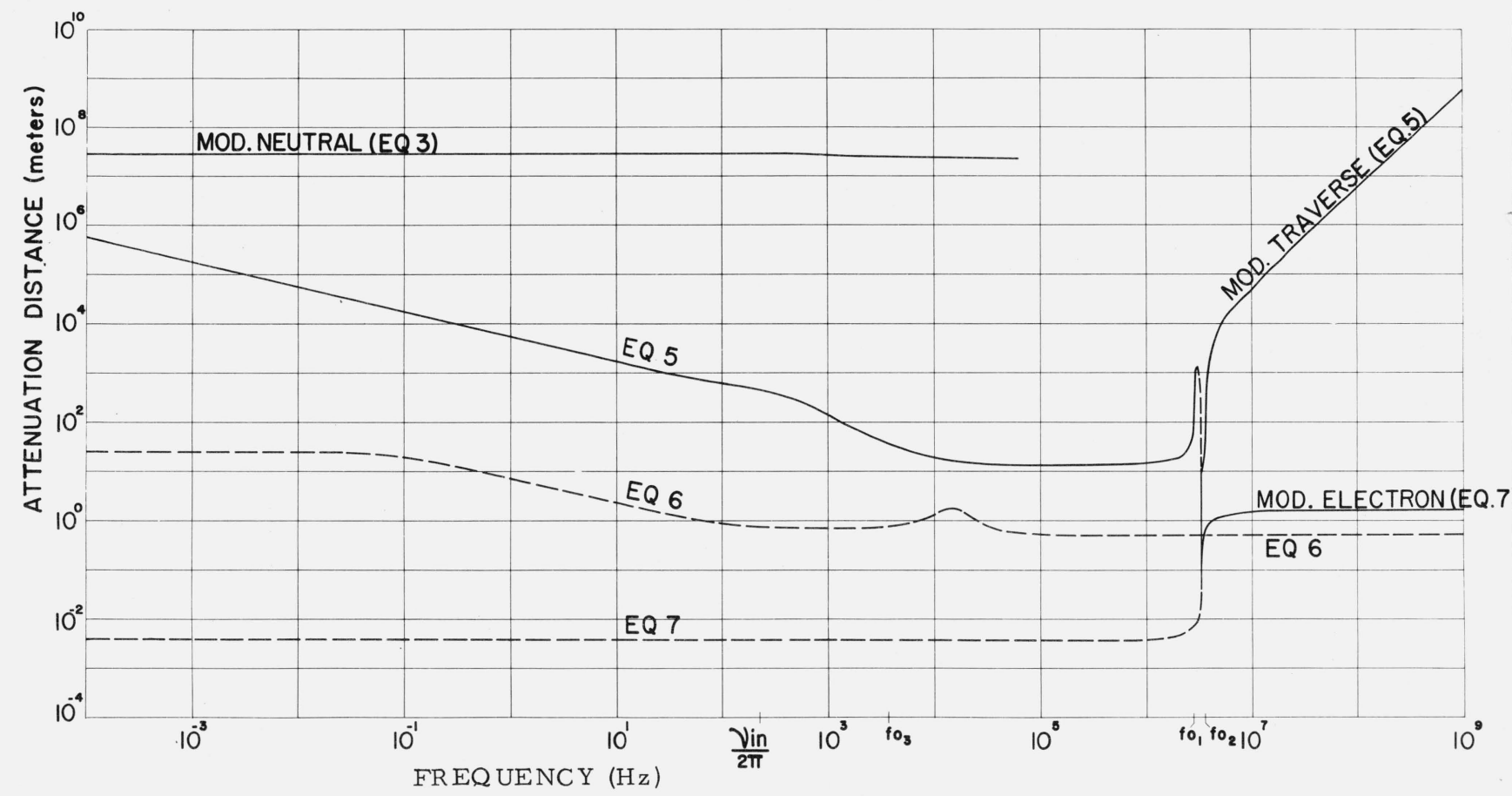

FiguRE 6. Attenuation distances as a function of frequency for waves traveling perpendicular to a uniform magnetic field at $100 \mathrm{~km}$. 


\begin{tabular}{|c|c|c|c|c|}
\hline \multirow{3}{*}{ 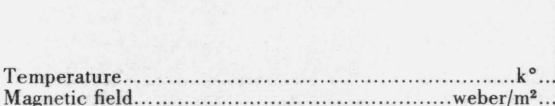 } & \multirow{3}{*}{$\begin{array}{l}T \\
B_{0}\end{array}$} & $80 \mathrm{~km}$ & $100 \mathrm{~km}$ & $300 \mathrm{~km}$ \\
\hline & & 205 & 280 & 1428 \\
\hline & & $3.0 \times 10^{-5}$ & $3.0 \times 10^{-5}$ & $2.75 \times 10^{-5}$ \\
\hline 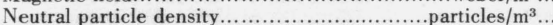 & $N_{1}$ & $4.38 \times 10^{20}$ & $5.4 \times 10^{18}$ & $1.035 \times 10^{15}$ \\
\hline 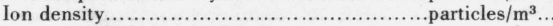 & $N_{0}$ & $1.0 \times 10^{9}$ & $1.3 \times 10^{11}$ & $1.8 \times 10^{12}$ \\
\hline 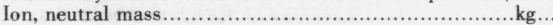 & $m_{i}$ & $4.82 \times 10^{-26}$ & $4.32 \times 10^{-26}$ & $3.570 \times 10^{-26}$ \\
\hline Electron plasma freq....................................ad/sec.. & $\omega_{e}$ & $1.783 \times 10^{6}$ & $2.033 \times 10^{7}$ & $7.568 \times 10^{7}$ \\
\hline 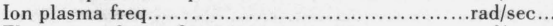 & $\omega_{i}$ & $7.754 \times 10^{3}$ & $9.339 \times 10^{4}$ & $3.822 \times 10^{5}$ \\
\hline 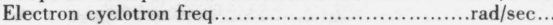 & $\omega_{c}^{e}$ & $5.276 \times 10^{6}$ & $5.276 \times 10^{6}$ & $4.836 \times 10^{6}$ \\
\hline 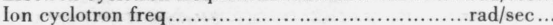 & $\omega^{i}$. & $9.97 \times 10^{1}$ & $1.112 \times 10^{2}$ & $1.234 \times 10^{2}$ \\
\hline Electron-neutral collision freq.......................... $\mathrm{sec}^{-1}$. & $\nu_{e n}$ & $6.517 \times 10^{6}$ & $9.39 \times 10^{4}$ & $4.064 \times 10^{1}$ \\
\hline Electron-ion collision freq $\ldots \ldots \ldots \ldots \ldots \ldots \ldots \ldots \ldots \ldots \ldots \mathrm{sec}^{-1}$. & $\nu_{e i}$ & $1.073 \times 10^{1}$ & $8.743 \times 10^{2}$ & $1.051 \times 10^{3}$ \\
\hline Ion-neutral collision freq................................. $\sec ^{-1}$. & $\nu_{\text {in }}$ & $1.022 \times 10^{5}$ & $1.406 \times 10^{3}$ & $3.261 \times 10^{-1}$ \\
\hline Neutral-electron collision freq.............................. $\sec ^{-1}$.. & $\nu_{n e}$ & $2.811 \times 10^{-10}$ & $4.766 \times 10^{-8}$ & $1.803 \times 10^{-6}$ \\
\hline 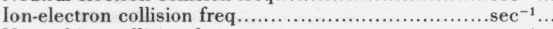 & $\nu_{i e}$ & $2.028 \times 10^{-4}$ & $1.843 \times 10^{-2}$ & $2.681 \times 10^{-2}$ \\
\hline Neutral-ion collision freq................................ $\sec ^{-1} .$. & $\nu_{n i}$ & $2.334 \times 10^{-7}$ & $3.385 \times 10^{-5}$ & $5.672 \times 10^{-4}$ \\
\hline & $\omega_{0}$ & $5.569 \times 10^{6}$ & $2.101 \times 10^{7}$ & $7.583 \times 10^{7}$ \\
\hline & $\omega_{01}$ & $5.465 \times 10^{5}$ & $1.787 \times 10^{7}$ & $7.330 \times 10^{7}$ \\
\hline & $\omega_{02}$ & $5.822 \times 10^{6}$ & $2.314 \times 10^{7}$ & $7.814 \times 10^{7}$ \\
\hline & & $7.346 \times 10^{3}$ & $2.345 \times 10^{4}$ & $2.438 \times 10^{4}$ \\
\hline Ion-neutral sound speed................... & $U_{i, n}$ & $3.12 \times 10^{2}$ & $3.86 \times 10^{2}$ & $9.6 \times 10^{2}$ \\
\hline 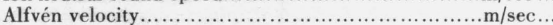 & $V_{A}$ & 5.83 & $5.53 \times 10^{1}$ & $4.02 \times 10^{3}$ \\
\hline 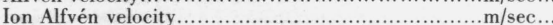 & $V_{A}^{\prime}$ & $3.86 \times 10^{6}$ & $3.57 \times 10^{5}$ & $9.68 \times 10^{4}$ \\
\hline 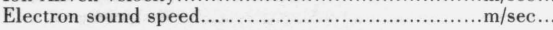 & $U_{e}^{A}$ & $7.2 \times 10^{4}$ & $8.4 \times 10^{4}$ & $1.895 \times 10^{5}$ \\
\hline
\end{tabular}

From the $300 \mathrm{~km}$ curves it can be seen that the phase velocity starts out at the Alfvén velocity, $V_{A}$, for $\omega<<\nu_{n i}$, and increases to the ion Alfvén velocity, $V_{A}^{\prime}$, for $\nu_{i n}<<\omega<<\omega_{c}^{i}$. Just below the ion cyclotron frequency, $\omega_{c}^{i}$, the LCP wave and the RCP wave separate. The LCP wave resonates at $\omega_{c}^{i}$, and is strongly damped for frequencies between $\omega_{c}^{i}$ and $\omega_{01}=\left[\omega_{e}^{2}\right.$ $\left.+\left(\frac{1}{2} \omega_{c}^{e}\right)^{2}+\omega_{c}^{e} \omega_{c}^{i}\right]^{1 / 2}-\frac{1}{2} \omega_{c}^{e}$. The phase velocity in this frequency band is high, since $k$ is almost purely imaginary.) Above $\omega_{01}$, the attenuation is again slight, and the phase velocity decreases towards the speed of light, $c$, for $\omega>>\omega_{01}$. The RCP wave resonates at $\omega_{c}^{e}$ and is strongly damped for frequencies between $\omega_{c}^{e}$ and $\omega_{02}=\omega_{01}+\omega_{c}^{e}$; then, above $\omega_{02}$ the attenuation is again slight, and the phase velocity decreases towards $c$, for $\omega>>\omega_{02}$. The phase velocity in the strongly damped frequency bands, $\omega_{c}^{i}<\omega<\omega_{01}$ for the LCP and $\omega_{c}^{e}<\omega<\omega_{02}$ for the RCP waves, is infinite $(\operatorname{Re} k=0)$ in the limit of no collisions. That the phase velocity is large but finite at $300 \mathrm{~km}$ is a consequence of small but nonzero collision frequencies.

On the $100 \mathrm{~km}$ curves there is no resonance at $\omega_{c}^{i}$, the LCP and RCP waves have widely separated phase velocities and attenuation distances, for all save very high frequencies, and the phase velocity never levels off at $V_{A}$ or $V_{A}^{\prime}$. This occurs because $\nu_{i n}>>\omega_{c}^{i}$. while $\nu_{e}<<\omega_{c}^{e}$. Inspection of the quantity in brackets in the dispersion relation shows for a wide range of frequencies $\left(\omega_{c}^{i} \rho_{i} / \rho_{n}<\omega<\omega_{c}^{e}\right)$, that $\pm \omega_{c}^{e} / \omega$ is the largest term, which accounts for the wide split in the LCP and RCP waves and the absence of the resonance at $\omega_{c}^{i}$. It is this term, too, which is responsible for the steady increase in the phase velocity, since $k$ is proportional to $\omega^{1 / 2}$; hence the phase velocity is proportional to $\omega^{1 / 2}$. Although the resonance at $\omega_{c}^{i}$ is absent, the attenuation distance is very small for the LCP wave between $\omega_{c}^{i}$ and $\omega_{01}$ and the RCP wave between $\omega_{c}^{e}$ and $\omega_{02}$ as in the $300 \mathrm{~km}$ case. For $\omega_{c}^{i}<<\nu_{i n}$, the "magneto-hydrodynamic" term, $\omega_{c}^{e} \omega_{c}^{i} / \omega^{2} f^{2}$, does not dominate until $\omega<<\omega_{c}^{i}\left(\rho_{i} / \rho_{n}\right)$, which is well below the smallest frequency used in our calculations. The phase velocity would, however, approach $V_{A}$ for both the LCP and RCP waves if the calculations were extended to these very low frequencies.

At $80 \mathrm{~km}$ the collisional terms in the dispersion relation are still more important, since $\omega_{c}^{i}<<\nu_{i n}$ and $\omega_{c}^{e}$ and $\nu_{e}$ are of comparable magnitude. Hence, for $\omega_{c}^{i} \rho_{i} /$ $\rho_{n}<<\omega<<\omega_{c}^{e}$, the expression in brackets approximately equals $\left( \pm \omega_{c}^{e}+i \nu_{e}\right) / \omega$. Thus the LCP and RCP curves are separated, but not as much as in the $100 \mathrm{~km}$ case, there is no resonance at $\omega_{c}^{i}$ or $\omega_{c}^{e}$, and the phase velocity, over a wide range of frequencies increases at a rate proportional to $\omega^{1 / 2}$.

The remaining figures all show the phase velocities and attenuation distances for the four coupled longitudinal and transverse waves able to propagate perpendicular to the magnetic field. The situation is complicated by the fact that several approximate dispersion equations have to be pieced together according to the scheme outlined in table 1 of Tanenbaum and Mintzer [1962]. Moreover, although there are four solutions to the dispersion equation, one or more of these usually represents a highly attenuated wave. Hence, the solid and broken curves in figures 3 to 8 represent, respectively, waves with large or small attenuation distances.

Although the terminology cannot be used very precisely, we can classify the waves with large attenuation distances as "modified" neutral, ion, electron, or transverse waves. The modified neutral wave is described by the dispersion relation

$$
k^{2} U_{n}^{2}=\omega^{2}(1+\epsilon), \quad(\text { for }|\epsilon|<<1),
$$

with

$$
\begin{aligned}
\epsilon & =\frac{i \nu_{n i}}{\omega}\left[\frac{\nu_{i n} X+i \omega}{\nu_{i n}(1+X)+2 \nu_{n i}+i \omega}\right], \\
X & =\omega_{c}^{e} \omega_{c}^{i} / \nu_{e} \nu_{i n} .
\end{aligned}
$$


For a weakly ionized gas (3) can be used (i.e., $|\epsilon|$ is small) provided $\omega$ exceeds $\nu_{n i} X /(1+X)$. Since this is an extremely low frequency, (3) could be applied for our entire range of $\omega$. As shown in the figures this modified neutral wave has a phase velocity of $U_{n}$ and an attenuation distance which decreases from $2 U_{n}(1+X) / \nu_{n i} X$, for low frequencies, to $2 U_{n} / \nu_{n i}$, for high frequencies. At $300 \mathrm{~km} X \cong 10^{7}$; consequently there is no noticeable difference. At $100 \mathrm{~km} X \cong 4$, so the change is slight. At $80 \mathrm{~km} X \cong 10^{-3}$, so the attenuation distance increases by about $10^{3}$.

The behavior of the remaining curves is determined in large measure by the single "high phase velocity" dispersion relation

$k^{2} c^{2}=\omega^{2}-\omega_{e}^{2}\left[1-\frac{\omega_{c}^{e} \omega_{c}^{i}}{\omega^{2} f^{2}}+\frac{i \nu_{e}}{\omega}\right.$

$$
\left.-\frac{\left(\omega_{c}^{e}\right)^{2}}{\omega^{2}-\omega_{e}^{2}+i \nu_{e} \omega-\left(\omega_{c}^{e} \omega_{c}^{i} / f^{2}\right)}\right]^{-1}
$$

which is valid for $\left|\omega^{2} / k^{2}\right|>>U_{n}^{2}$. When collisional damping effects are slight, as at $300 \mathrm{~km}$, the phase velocity obtained from (5) starts out at the Alfvén velocity, $V_{A}$, for $\omega<<\nu_{n i}$ and increases to $V_{A}^{\prime}$ for $\nu_{i n}$ $<<\omega<<\omega_{03}=\omega_{i}\left(\omega_{c}^{e} \omega_{c}^{i}\right)^{1 / 2} /\left(\omega_{i}^{2}+\omega_{c}^{e} \omega_{c}^{i}\right)^{1 / 2}$. At $\omega_{03}$ this wave resonates and continues on for higher frequencies as a modified ion wave described by the dispersion relation

$$
k^{2} U_{i}^{2}=\frac{1}{2}\left(\Omega^{2}-\Omega_{c}^{e} \Omega_{c}^{i}\right)-\Omega_{i}^{2}+\left[\frac{1}{4}\left(\Omega^{2}+\Omega_{c}^{e} \Omega_{c}^{i}\right)^{2}+\Omega_{i}^{4}\right]^{1 / 2},
$$

with $\Omega^{2}=\omega^{2}+i \omega \nu_{i n}, \Omega_{c}^{e, i}=\omega_{c}^{e, i}\left[1+i\left(\nu_{e} / \omega\right)\right]^{-1 / 2}$, and $\Omega_{i}^{2}=\omega_{i}^{2}+i \omega \nu_{e}\left(m_{e} / m_{i}\right)$. (For frequencies below $\omega_{03}$, the attenuation distance obtained from (6) is always very short.)

For $\omega>\omega_{03}$ the attenuation distance computed from (5) is very short and the phase velocity very large until $\omega$ reaches the first cutoff frequency, $\omega_{01}$; then, for $\omega_{01}<\omega<\omega_{0}=\left[\omega_{e}^{2}+\left(\omega_{c}^{e}\right)^{2}\right]^{1 / 2}$, the attenuation distance is again large and the phase velocity decreases toward c. At $\omega_{0}$ there is a second resonance, and the wave continues for higher frequencies as a modified electron wave described by the dispersion relation

$$
k^{2} U_{e}^{2}=\omega^{2}-\omega_{e}^{2}+i \nu_{e} \omega-\left(\Omega_{c}^{e}\right)^{2} .
$$

[For frequencies below $\omega_{0}$, the attenuation distance obtained from (7) is always short.] For $\omega>\omega_{0}$ (5) again describes a wave with very short attenuation distance until $\omega$ reaches the second cutoff frequency $\omega_{02}$. Thereafter this modified transverse wave is only slightly attenuated and has a phase velocity that decreases towards $c$ for $\omega>>\omega_{02}$.

All the features described above appear only in the $300 \mathrm{~km}$ curves shown in figures 3 and 4 . At $100 \mathrm{~km}$ (figs. 5 and 6) the phase velocity obtained from (5) does reach $V_{a}^{\prime}$ for $\nu_{i n}<\omega<\omega_{03}$, but the resonance at $\omega_{03}$ is absent, although the attenuation distance is still short for $\omega_{03}<\omega<\omega_{01}$. This occurs since $\nu_{e}$ exceeds $\omega_{03}$; hence the term $i \nu_{e} / \omega$ dominates the bracketed expression in (5) near $\omega_{03}$. In addition the ion wave [as described by (6)] is greatly attenuated for all $\omega$, since its attenuation distance, which approaches $2 U_{i} / \nu_{\text {in }}$ for large $\omega$, falls from $6,000 \mathrm{~m}$ at $300 \mathrm{~km}$ to $55 \mathrm{~cm}$ at $100 \mathrm{~km}$ and $6.2 \mathrm{~mm}$ at $80 \mathrm{~km}$.

At $80 \mathrm{~km}$ (figs. 7 and 8) collisions are of still greater importance in (5), since $\nu_{\text {in }}$ exceeds $\omega_{03}$, eliminating the possibility of Alfvén waves with phase velocity $V_{A}^{\prime}$; and $\nu_{e}$ exceeds $\omega_{0}$, eliminating the resonance at $\omega_{0}$. The term $i \nu_{e} / \omega$ now dominates the term in brackets in (5) for a wide range of frequencies, resulting in curves that are almost identical to the LCP and RCP waves of figures 1 and 2 at $80 \mathrm{~km}$. In addition, the modified electron wave [as described by (7)] is greatly attenuated for all $\omega$, since its asymptotic attenuation distance, $2 U_{e} / \nu_{e}$, falls from $380 \mathrm{~m}$ at $300 \mathrm{~km}$ and $1.8 \mathrm{~m}$ at $100 \mathrm{~km}$, to $2.2 \mathrm{~cm}$ at $80 \mathrm{~km}$.

\section{References}

Chapman, S. (1956), The electrical conductivity of the ionosphere: A review, Nuovo Cimento 4, 1385.

Cowling, T. G. (1945), The electrical conductivity of an ionized gas in a magnetic field with applications to the solar atmosphere and ionosphere, Proc. Roy. Soc. (London) 183A, 453.

Minzner, R. A., K. S. W. Champion, and H. L. Pond (1959), The ARDC model atmosphere, 1959 AFCRC TR-59-267 (Geophysics Research Directorate, Air Force Cambridge Research Center, Bedford, Mass.).

Tanenbaum, B. S., and D. Mintzer (1962), Wave propagation in a partly ionized gas, Phys. Fluids 5, 1226.

Tanenbaum, B. S., and D. Mintzer (1963), Wave propagation with a transverse magnetic field, Proc. Intern. Conf. Ionosphere, London, July 1962 (Inst. Phys. Soc. London). 